\title{
Tipicità delle costruzioni presentative per l'italiano neostandard
}

\section{Abstract}

The aim of this paper is to examine the distribution of presentative constructions in contemporary Italian and to verify whether their occurrence depends on language external factors. Presentative constructions have been studied particularly by Italian linguists during the Eighties, and were defined as being 'typical' of so-called Neostandard Italian. The analysis is based on three corpora of contemporary Italian, viz. two oral corpora (LIP and CLIPS) and one written corpus (CORIS/CODIS).

Counter to expectations, presentative constructions are far from frequent in contemporary Italian and therefore cannot be considered as being 'typical' of Neostandard Italian. They are, however, attested both in written and oral Italian, with a clear preference for the latter context, in which they are evenly distributed in formal and informal registers. We conclude by discussing the differences between the linguistic phenomena traditionally included into the 'feature pool' of Neostandard Italian.

Keywords: linguistics, Neostandard Italian, presentative constructions, syntax, variation

\section{Introduzione}

Nei centocinquant'anni della storia dell'italiano postunitario, la fisionomia sociolinguistica italiana ha subito profondi mutamenti: si è passati dall'italiano prevalentemente scritto e riservato quasi esclusivamente ai ceti più istruiti (De Mauro 1963), a un italiano parlato, a volte lontano dalla norma tradizionale e caratterizzato dal riemergere di tendenze linguistiche antiche, tradizionalmente tenute fuori dalla codificazione della buona lingua (D'Achille 1990). Tale processo di ri-standardizzazione è stato inizialmente rilevato e descritto in alcuni ormai classici studi pubblicati negli anni Ottanta. In questi lavori veniva individuata una nuova varietà di lingua, centrale nel repertorio linguistico italiano: l'italiano dell'uso medio (Sabatini 1985) o italiano neostandard (Berruto 1987) o anche italiano tendenziale (Mioni 1983). A prescindere da queste differenze terminologiche ${ }^{1}$, vari linguisti hanno riconosciuto in questa varietà un'emergente lingua panitaliana, in uso sia nel parlato che nello scritto informale e mediamente formale, dagli italofoni di classe «medio-alta» (Sobrero 1992). Dagli anni Ottanta in poi, molti studiosi si sono dedicati ad approfondire questo processo di ri-standardizzazione, analizzandone in particolare i tratti fonetici, fonologici, morfosintattici e lessicali più ricorrenti da un punto di vista descrittivo e/o più spiccatamente teorico. Un'ampia e aggiornata rassegna degli studi sulle dinamiche interne al repertorio linguistico italiano è presentata in Cerruti (in corso di stampa).

Ricerche recenti svolte su fenomeni ritenuti prototipici del neostandard hanno contribuito a definire in maggior dettaglio le caratteristiche di tale varietà. Colpiscono in questo filone alcune

\footnotetext{
${ }^{1}$ Le scelte terminologiche di Sabatini («dell’uso medio», Sabatini 1985) e Berruto («neostandard», Berruto 1987) sottolineano rispettivamente l'ampia convergenza della comunità linguistica su questa varietà linguistica e la funzione di nuova norma di riferimento che nel corso degli anni è venuta ad assumere. Con il termine «tendenziale», Mioni (1983) evidenzia invece la 'tendenza' dei parlanti con competenze meno avanzate ad avvicinarsi alla norma di prestigio.
} 
indagini che mostrano la scarsa diffusione di certi tratti inizialmente definiti «diagnostici» (Berruto 1987) dell'italiano medio. Ad esempio, diversi studi (Alfonzetti 2002; Aureli 2003, 2005), sull'uso non standard del pronome relativo che, hanno illustrato che si tratta di un uso tipico del parlato substandard e per nulla preponderante nel parlato colloquiale. Parimenti, in un contributo recente, Crocco (2010) ha evidenziato come un fenomeno apparentemente «comune» quale la dislocazione a destra sia diffuso diversamente in diatopia. Infine, Roggia $(2008,2008$ a) ha dimostrato che le costruzioni scisse (del tipo È la nebbia che mi fa paura / Era questo a cui ti riferivi?), contrariamente a quanto presupposto in studi precedenti, sono nettamente più frequenti nello scritto o in contesti di lingua parlata formale. Questi risultati suggeriscono che i fenomeni del neostandard, pur rappresentando importanti risorse linguistiche, non sono sempre tratti comuni o medi e che possono essere marcati in senso diamesico, diastratico, diafasico o diatopico. Emerge quindi la domanda relativa a quale sia l'effettivo peso dei tratti individuati nei primi studi nel definire la varietà neostandard.

Nel presente lavoro ci si propone di riprendere e approfondire queste idee analizzando la diffusione di un altro fenomeno considerato tipico dell'italiano neostandard: le costruzioni presentative articolate in due clausole, di cui la prima consistente in un sintagma nominale (SN) introdotto da $c^{\prime} e^{\prime} / c i$ sono e la seconda in una (pseudo)relativa introdotta da che. Lo studio ha lo scopo duplice di descrivere la distribuzione di queste costruzioni in un campione di italiano contemporaneo e di cercare di determinare da quali fattori dipenda l'occorrenza della costruzione in questione nei campioni di lingua esaminati. Attraverso l'analisi della costruzione presentativa, con questo lavoro s'intende pertanto contribuire a una definizione più accurata della varietà neostandard rispetto alle sue variazioni diatopiche, diafasiche e diamesiche.

La scelta di prendere in esame le costruzioni presentative nasce da alcune costatazioni. In primo luogo, nonostante si tratti di un costrutto osservato già negli anni Ottanta quale fenomeno tipico dell'italiano contemporaneo (Sabatini 1980, 1982; Berruto 1987), esso ha suscitato ancora poco interesse scientifico a livello nazionale e internazionale. Infatti, tra i lavori più importanti dedicati interamente alle costruzioni presentative in italiano, si possono citare solo Berruto (1986), Berretta (1995), De Cesare $(2006,2007)$ e Venier $(2002,2004)$. Altri lavori accennano al fenomeno nell'ambito di uno studio più ampio delle costruzioni introdotte da c'è con valore esistenziale o locativo (Berretta 1995a; Panunzi 2005; De Cesare 2010; Cruschina 2012) o in prospettiva storico-tipologica (Blasco Ferrer 2004). Neppure le grammatiche italiane vi dedicano molta attenzione: a parte alcuni cenni all'esistenza di questo tratto detto «tipico» del parlato (Serianni 2000; si veda anche Simone et. al. 2011, con cenni alla costruzione presentativa per esempio in Lombardi Vallauri 2011), sono quasi inesistenti gli approfondimenti sull'uso o sulle sue proprietà linguistiche e le funzioni testuali proprie del costrutto. Anche a livello internazionale sono rari gli studi sull'italiano riguardanti costruzioni del tipo c'èlci sono ... che. Maggiore, benché sempre limitata, attenzione hanno destato invece le analoghe costruzioni in francese (avoir-clefts; si vedano: Ashby 1995, Béguelin 2003 e Choi-Jonin \& Lagae 2005) e in inglese (there-clefts; si vedano: Lambrecht 1988, 2001 e Davidse 1999). In secondo luogo, 
e in linea con gli obiettivi di questo studio, l'esame delle costruzioni scisse ci pare interessante anche per motivi variazionali. Benché Berruto (1986) avesse già sollevato, in uno dei primi studi sul c'è presentativo $^{2}$, l'ipotesi che la ricorrenza di tale costruzione fosse suscettibile a variazioni diatopiche, il fenomeno non è mai stato oggetto di uno studio su corpus. Infine, l'interesse per le costruzioni presentative scaturisce proprio dalla loro definizione come tratto saliente dell'italiano parlato contemporaneo e in particolare del neostandard (Berruto 1986; Sabatini 1980, 1982). Con questo terzo argomento ci si riallaccia allo scopo generale del presente studio, che è quello di verificare l'effettivo peso attuale dei fenomeni che sono stati considerati come «nucleo» del neostandard.

L'analisi proposta in questo lavoro poggia su tre corpora d'italiano contemporaneo, parlato corpus LIP (De Mauro et al. 1993) e corpus CLIPS (Albano Leoni 2006) - e scritto (corpus CORIS/CODIS, Rossini Favretti 2000). Nelle pagine seguenti ne sarà illustrata la composizione.

Prima di entrare in argomento, saranno brevemente descritte e delimitate le costruzioni presentative trattate in queste pagine ( $(2)$; quindi saranno discussi i corpora utilizzati ( $(3)$. Nel paragrafo successivo ( $\$ 4)$ si daranno i dati relativi alla frequenza e alla distribuzione variazionale (diatopica, diafasica e diamesica) delle costruzioni. Come si vedrà, la costruzione è attestata in tutta l'Italia, benché in maniera disomogenea, e in particolare nel parlato di vari registri. I risultati sono quindi riassunti e confrontati con quelli di alcuni studi precedenti sullitaliano neostandard $(\S 5)$. Nell'ultimo paragrafo $(\S 6)$ saranno formulate alcune conclusioni che iscrivono i risultati nel quadro generale del rapporto tra varietà parlate e varietà neostandard.

\section{Le costruzioni presentative}

In questo studio sono prese in esame le costruzioni presentative, spesso illustrate dall'ormai famoso esempio che dà titolo al contributo di Berretta (1995) C'è il gatto che ha fame. Si tratta del costrutto introdotto da $c^{\prime}$ 'è (o al plurale ci sono) seguito da un gruppo nominale o un pronome e da una pseudorelativa introdotta da che non flesso.

Dalla bibliografia sulle costruzioni italiane introdotte da c'èlci sono si ricavano classificazioni abbastanza eterogenee che non sempre sono in accordo tra di loro. Secondo Berruto 1986 e De Cesare 2007, le diverse costruzioni con c'è rappresentano sottotipi della categoria generale del ci presentativo. Di seguito si riportano i cinque tipi presentati nello studio di ambedue gli autori:
(1) c'è esistenziale: C'è la provvidenza.
(2) c'è locativo-deittico: Al Polo Nord ci sono gli orsi bianchi.
(3) c'è eventivo, di accadimento: Ci fu una disgrazia.
(4) c'è di presenza, datità: Ci sono due nuovi professori di romanistica.
(5) c'è presentativo: C'è un signore che vuole parlare con te.

\footnotetext{
${ }^{2} \ll$ Ho trovato per esempio molti più casi nei racconti dei reduci emiliano-romagnoli di Era come a mietere che non in quelli dei reduci lombardi de La Grande Guerra. La cosa non dovrebbe essere effetto distorto di interventi redazionali nella trascrizione in questo secondo caso, dato che il costrutto appare del tutto grammaticale, e corretto, anche nello scritto.» (Berruto 1986: 64 e 72-73).
} 
De Cesare (2007) osserva che solo l'esempio (5) illustra il vero e proprio costrutto presentativo, di cui un altro esempio è il già citato $C^{\prime}$ è il gatto che ha fame. Gli altri riguardano la categoria più generale, in cui il c'è ha un valore semantico-pragmatico diverso. Negli esempi da (1) a (4), infatti, si tratta di un valore semanticamente «pieno» di c'è (nelle parole di Berruto, il c'è qui ha un valore «esistenziale», «locativo-deittico», «eventivo» e «di presenza»), in (5) invece, il c'è ha un valore detto «vuoto» o grammaticalizzato (Berretta 1995; De Cesare 2007) e acquisisce un valore prettamente funzionale piuttosto che lessicale. Infatti, la frase C'è il gatto che ha fame, come affermano Berretta (1995) e De Cesare (2007), in linea con gli studi di Lambrecht (1994), serve a introdurre un nominale nuovo nell'universo del discorso, presentandolo in posizione focale. Per questo motivo l'autrice propone il termine «c'è focalizzante» per le costruzioni introdotte dal c'è a valore zero (De Cesare 2007). A livello sintattico, le due costruzioni si distinguono per il tipo di relativa che segue: le frasi introdotte dal c'è focalizzante vengono obbligatoriamente seguite da una pseudorelativa (con che), mentre le altre costruzioni non sempre sono seguite da una relativa $\mathrm{e}$, se lo sono, la relativa è restrittiva o appositiva (De Cesare 2007). La presenza della pseudorelativa è quindi considerata una caratteristica essenziale del costrutto presentativo.

Nonostante l'apparente somiglianza, la costruzione pseudorelativa ha proprietà differenti rispetto alle relative propriamente dette (Cinque 1988, Radford, 1975, Venier 2007). Di particolare rilievo per l'esame dei costrutti presentativi è il fatto che la pseudorelativa, a differenza della relativa appositiva e di quella restrittiva, ha una funzione predicativa piuttosto che modificativa ed anzi esprime la predicazione principale dell'enunciato, riferita all'antecedente precedentemente introdotto. Antecedente e pseudorelativa sono quindi in un rapporto di predicazione (Sornicola 2007, Venier 2007). La distinzione tra pseudorelativa e relativa restrittiva diventa problematica nel caso in cui l'antecedente della subordinata sia indefinito. Secondo De Cesare (2007), l'antecedente del costrutto con c'è focalizzante è tipicamente un SN "definito nel contesto di enunciazione", solitamente un nome proprio oppure un $\mathrm{SN}$ definito, come in:

(6) C'è Maria che dorme (De Cesare 2007).

Nel caso di un enunciato analogo ma con SN indefinito (come ad esempio: c'è un gatto che dorme) il confine tra pseudorelativa (con valore predicativo) e relativa restrittiva (con valore modificativo) non è netto e può dipendere dal contesto in cui l'enunciato occorre. Si noti però che la definitezza del SN introdotto da c'è non è ritenuta una caratteristica fondamentale del costrutto presentativo da tutti gli autori che si sono occupati dell'argomento. Ad esempio, in un recente lavoro, Cruschina (2012) distingue tra le costruzioni con c'è aventi valore locativo e quelle con valore esistenziale, identificandole in primo luogo in base al grado di definitezza del SN in esse contenuto: mentre nelle costruzioni esistenziali di norma compare un nominale indefinito (c'è un gatto), ciò non 
avviene nelle costruzioni locative, in cui invece il nominale tende ad essere definito (c'è Gianni). A dispetto della somiglianza, queste costruzioni hanno secondo l'autore strutture differenti, in quanto il pronome $c i$ è nel caso delle locative un elemento predicativo con valore deittico, mentre nel caso delle esistenziali ha valore di soggetto espletivo. In aggiunta, Cruschina osserva che le costruzioni presentative, caratterizzate dalla presenza di un elemento predicativo aggettivale o pseudorelativo che segue il SN introdotto da c'è, si collocano in una posizione intermedia tra le locative e le esistenziali. Tali costruzioni non sono soggette in italiano a restrizioni di definitezza, per cui il SN introdotto da $c^{\prime} \grave{e}$ che compare in posizione di focus può essere tanto definito quanto indefinito. L'autore afferma anche che, in queste costruzioni, entrano solitamente SN definiti oppure indefiniti specifici, mentre gli indefiniti non specifici, benché non impossibili, sono meno frequenti. In breve, mentre tutti sono d'accordo nel riconoscere in una costruzione con nome proprio e pseudorelativa (del tipo c'è Maria che dorme) un caso di costruzione presentativa, costruzioni analoghe contenenti un SN indefinito seguito da una clausola interpretabile come relativa restrittiva pongono maggiori problemi classificatori. Per ovviare a tali problemi, in questo lavoro, le costruzioni presentative sono state distinte da altri costrutti formalmente simili principalmente in base alle proprietà informative e funzionali ricordate poco fa. È stato quindi considerato presentativo un enunciato biclausale, in cui la prima clausola, aperta da c'è, introduce un $\mathrm{SN}$, definito o indefinito, che viene presentato in posizione post-verbale focale ma che funziona come topic della predicazione espressa dalla clausola (pseudo)relativa seguente. La (pseudo)relativa rappresenta quindi il fulcro predicativo dell'enunciato. Dall'analisi sono stati invece esclusi i casi in cui un costrutto introdotto da c'è presentasse nella seconda parte una clausola relativa con valore chiaramente appositivo, nonché i casi in cui la struttura c'è...che solo apparentemente fosse del tipo esaminato in queste pagine (es.: costruzioni locative o costruzioni in cui la relativa non ha come antecendente il SN introdotto da $c$ 'è). I seguenti enunciati tratti da CLIPS e dal LIP esemplificano casi non inclusi nell'analisi ${ }^{3}$ :

(7) e _ poi c'è il pollice_che è spostato (CLIPS Firenze DGTDB04F)

(8) ah! C'è il puntino nero _ che <ee > a te non sta alla sinistra di una farfalla, tanto per dirne una per esempio (CLIPS Napoli DGMTA01N)

(9) C: poi c'è poi c'è il tranello_che è per esempio

A: l'inganno (LIP ROMA C9)

(10) poi c'è_un piccolo schermo con una donnina che canta _ con la bocca aperta (CLIPS, Firenze DGTDA01F)

Di seguito sono riportati alcuni esempi di enunciati tratti dai corpora CLIPS e LIP che sono stati invece inclusi nel campione analizzato per questo lavoro:

(11) B: ah chiamavano anche te marescialla

\footnotetext{
${ }^{3}$ La trascrizione ortografica fornita negli esempi è semplificata rispetto a quella di cui sono corredati i testi del corpus CLIPS. Pause, inspirazioni ecc. sono state rese in questa trascrizione con un trattino in basso "_". I commenti e le indicazioni riguardanti sovrapposizioni e fenomeni vocali sono stati eliminati dalla trascrizione.
} 
G: mi chiamava marescialla qualcuno

B: ah ecco eh perché appunto giocando sul nome uno_ pensa mentre invece marescialla proprio_ perché Pino è diventato un soldatino ubbidiente fedele come un carabiniere fedele nei secoli ah c'è la signorina che entra

I: a Radio Firenze sono le ore ventitre l'ora Imperia la catena di negozi che ti fa [interruzione] (LIP FIRENZE E15)

(12) A: [è meglio] ricordarlo sempre il caso Tortora soprattutto quando si parla di droga soprattutto quando si parla di droga

B: ci sono c’è Nino Petrone del Messaggero che vuol rispondere all'avvocato prego (LIP NAPOLI E8)

(13) c'è la signora Barbara che invece chiama da Padova (LIP MILANO E6)

(14) quindi eh appunto in tutte le fiabe tutti i ragazzi c'è un ragazzo che deve superare delle prove delle prove per diventare adulto (LIP ROMA C9)

(15) c'è Letizia che che dovrebbe andar via (LIP ROMA A5)

Questi enunciati, oltre a condividere la funzione discorsiva e la struttura pragmatica prima descritte, presentano anche le tre proprietà morfosintattiche che, secondo De Cesare (2007), permettono di distinguere gli impieghi prettamente presentativi (o 'focalizzanti') di c'è dagli altri: la possibilità di ricorrere in un impiego isolato (ossia senza la (pseudo)relativa), di invertire l'ordine dei costituenti e di negare o interrogare il costrutto introdotto da c'è. Riprendendo l'esempio (11) citato qui sopra (c'è la signorina che entra), è possibile verificare che le costruzioni presentative non rispondono a nessuno dei tre criteri, vale a dire: non possono ricorrere senza (pseudo)relativa perlomeno non senza perdere le loro proprietà semantico-funzionali di base (c'è la signorina). Nei casi in cui è assente la (pseudo)relativa (c'è la signorina) la costruzione viene ad esprimere la presenza "qui e ora" dell'entità cui il SN si riferisce, e assume quindi un valore locativo equivalente a quello espresso dall'enunciato La signorina è qui. Inoltre il costrutto presentativo non consente l'inversione dei costituenti (*La signorina c'è che entra) e non può essere negato né interrogato per chiedere informazioni ${ }^{4}$ (*Non c'è la signorina che entra e neppure *C'è la signorina che entra?).

\section{Composizione del corpus e precisazioni metodologiche}

Nei paragrafi che seguono saranno presentati dei dati sulla costruzione presentativa tratti da corpora di italiano parlato e scritto. Il lavoro ha in primo luogo lo scopo di fornire dati sulla diffusione delle costruzioni presentative in tre dimensioni variazionali. La diatopia e la diafasia si giustificano poiché l'italiano neostandard è stato definito in senso diatopico come comune en in senso diafasico come medio. L'interesse della dimensione diamesica deriva dal fatto che il c'è presentativo si è trovato spesso incluso fra le «costruzioni marcate», ritenute tipiche delle varietà ancorate al parlato.

Per questo lavoro sono stati selezionati tre corpora, di cui due d'italiano parlato, il LIP e il CLIPS, e uno di italiano scritto, il CORIS/CODIS. Il CLIPS e il LIP sono ben noti e largamente usati. Il corpus LIP è stato scelto per la sua forte stratificazione diafasica e diatopica. Sono state prese in considerazione tutte e quattro le località (Milano, Firenze, Roma, Napoli) e tutti i tipi di testo, sia quelli più informali $(\mathrm{A}$ e B) che quelli più formali $(\mathrm{C}, \mathrm{D}, \mathrm{E})$. Nel suo complesso, il corpus LIP

\footnotetext{
${ }^{4}$ È possibile tuttavia che un'interrogativa simile a c'è la signorina che entra? ricorra come domanda incredula o domanda di conferma, in un contesto in cui il parlante voglia controllare quanto affermato dall'interlocutore.
} 
contiene approssimativamente 500.000 parole. Del corpus CLIPS sono state selezionate solo le sezioni dialogiche (dialoghi map task e dialoghi del tipo «test delle differenze») relative alle città presenti nel LIP (Milano, Firenze, Roma, Napoli). Questi dati sono stati utilizzati per un confronto con il LIP, per le ragioni che saranno esposte più avanti (cfr. sezione 4.1).

Il corpus CORIS/CODIS è stato scelto per vari motivi: non solo consente di fare un paragone a livello diamesico con il LIP, ma è inoltre un corpus molto ampio (circa 100 milioni di parole grafiche, p.g.) che permette uno studio empirico su larga scala, con dati bilanciati e rappresentativi di diversi tipi testuali. Per questo lavoro è stata selezionata solo una parte della sezione «Stampa» (circa tre milioni di parole). Come mostrato da un recente studio di Bonomi (2002), la scrittura giornalistica è selettivamente permeabile ai tratti dell'uso medio, poiché tende ad accogliere quelli meno marcati, rigettando invece quelli più marcati in senso diamesico (e diastratico), come il che polivalente.

Tabella 1
LIP
CODIS - stampa
ca. 500.000 p.g.
ca. 3.000 .000 p.g.

L'estrazione ed etichettatura delle ricorrenze dei fenomeni è stata effettuata con il programma Abundantia Verborum (Speelman 1997). Per l'estrazione delle occorrenze del c'è presentativo, si è partiti dalle caratteristiche linguistiche precisate nel paragrafo 2 .

\section{Distribuzione delle costruzioni presentative nei corpora}

Lo spoglio ha registrato la presenza di 127 costruzioni con il c'è presentativo nel corpus LIP e 116 nel corpus CODIS. Nel complesso sono quindi state rilevate 243 occorrenze. Questi dati (cfr. tabella 2) confermano innanzitutto la bassa frequenza della costruzione nel campione esaminato e ci danno una prima idea di come il fenomeno sia distribuito nel parlato e nello scritto. Ricordiamo infatti che le dimensioni assolute del corpus CODIS, espresse in parole grafiche, superano di circa sei volte quelle del LIP:

Tabella 2

$$
\begin{array}{ll}
\text { LIP (500.000 p.g.) } & \text { CODIS STAMPA ( } 3 \text { mln. p.g.) } \\
127 & 116
\end{array}
$$

\subsection{Variabilità diatopica}

La caratterizzazione del costrutto presentativo in quanto tratto tipico dell' «italiano parlato unitario» (Sabatini 1980) invita innanzitutto a porsi il problema della diffusione geografica del fenomeno. Il corpus LIP, che raccoglie materiali registrati in quattro grandi città che rappresentano aree geografiche diverse, permette una verifica affidabile, poiché le sezioni diatopiche del corpus 
hanno pressoché lo stesso numero di parole grafiche (circa 125.000 ciascuna). Nella seguente tabella vengono riportati i risultati:

Tabella 3

$\begin{array}{llllll} & \text { Firenze } & \text { Roma } & \text { Napoli } & \text { Milano } & \text { Totale } \\ \text { C'è presentativo } & 38 / 127 & 42 / 127 & 23 / 127 & 24 / 127 & 127 \\ & \mathbf{3 0 \%} & \mathbf{3 3 \%} & \mathbf{1 8 \%} & \mathbf{1 9 \%} & \mathbf{1 0 0 \%}\end{array}$

Il costrutto è attestato in tutte le varietà campionate nel LIP, tuttavia in modo non uniforme. Infatti, emerge una differenza lieve ma significativa $(\mathrm{p} \leq 0,031)$ tra le ricorrenze registrate a Firenze e Roma, dove la costruzione appare abbastanza diffusa (con circa il 30\% delle occorrenze) e quelle registrate a Napoli e Milano, dove la presenza del c'è presentativo appare nettamente inferiore (18\% e 19\% dei casi). Per quanto riguarda la sezione di Napoli, era necessario verificare se e fino a che punto la bassa ricorrenza delle costruzioni con il c'è presentativo non fosse causata dall'esistenza e diffusione della variante regionale $c i$ sta, tipica delle varietà meridionali. A questo scopo sono state estratte e analizzate le occorrenze della variante regionale ci sta (nei tempi presente, imperfetto, futuro, passato prossimo e passato remoto) nell'intero corpus LIP. Le sole tre costruzioni presentative con stare ricavate nel LIP appaiono tutte nei testi napoletani:

(16) ci sta gente che chiede il certificato

(17) e che significa qua ci stanno gente che so' detenute da dieci quindici anni

(18) ci stanno alcune questioni che vanno secondo me vanno giudicate anche in questa direzione

Queste tre occorrenze non modificano i dati sulla distribuzione presentata nella tabella 4: la seguente tabella presenta i dati complessivi includendo le occorrenze di ci sta presentativo.

Tabella 4

$\begin{array}{llllll} & \text { Firenze } & \text { Roma } & \text { Napoli } & \text { Milano } & \text { Totale } \\ \text { C'è presentativo } & 38 / 130 & 42 / 130 & 26 / 130 & 24 / 130 & 130 \\ \% & \mathbf{2 9 \%} & \mathbf{3 2 \%} & \mathbf{2 0 \%} & \mathbf{1 8 \%} & \mathbf{1 0 0 \%}\end{array}$

Per approfondire ulteriormente questi risultati, si è deciso di procedere a un confronto dei dati del LIP con quelli del corpus CLIPS. Questo corpus, nonostante meno ampio del LIP, presenta una campionatura diatopica più stretta e controllata, poiché tutti gli informanti registrati nel corpus sono nati e cresciuti nelle quindici città scelte per la raccolta e sono inoltre figli di parlanti dello stesso centro urbano. Il LIP, invece, rappresenta le maggiori aree geografiche italiane attraverso le registrazioni effettuate, all'insaputa dei parlanti, nelle città di Milano, Firenze, Roma e Napoli, senza 
quindi possibilità di controllare puntualmente se gli informanti vi fossero effettivamente nati e/o cresciuti. Come già precisato nella parte metodologica (§3), del CLIPS sono stati selezionati solo i testi dialogici (map task e test delle differenze) per le quattro città del LIP. Nella tabella 5 sono confrontati i numeri assoluti e le percentuali di occorrenza delle costruzioni presentative nel corpus CLIPS:

$\begin{array}{llllll}\text { Tabella 5 } & & & & \\ & \text { Napoli } & \text { Roma } & \text { Milano } & \text { Firenze } & \text { Totale } \\ \text { C'è presentativo } & 7 / 14 & 5 / 14 & 2 / 14 & 2 / 14 & 14 \\ \% & \mathbf{5 0 \%} & \mathbf{3 5 \%} & \mathbf{1 4 \%} & \mathbf{1 4 \%} & \mathbf{1 0 0 \%}\end{array}$

La scarsa occorrenza del c'è presentativo nei testi selezionati del corpus CLIPS può essere spiegata tenendo conto delle tecniche di elicitazione con cui i dialoghi sono stati raccolti. I compiti utilizzati per queste tecniche sperimentali, consistenti nella ricostruzione di un percorso (map task) e nell'individuazione di piccole differenze tra due vignette (test delle differenze), influiscono infatti sul tipo di costruzioni sintattiche che si trovano nei dialoghi (Aureli, et al. 2005). Dato il tipo di giochi che i parlanti devono svolgere, quindi, non sorprende che siano più frequenti avverbi e costruzioni con un significato localizzante. Nel caso delle costruzioni con $c^{\prime}$ è qui in esame, più che le frasi presentative si osservano infatti frasi contenenti un c'è locativo-deittico, del tipo:

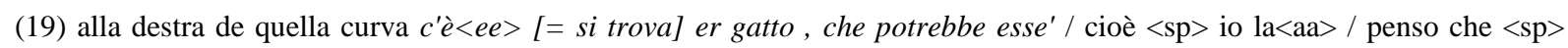
potrebbe esse' dest $+\langle\mathrm{pp}\rangle$ no $\langle\mathrm{sp}\rangle$ momento in cui tu $\langle\mathrm{sp}\rangle\langle\mathrm{eeh}\rangle$ con $+\langle\mathrm{sp}\rangle$ vai $\mathrm{s}+/$ stai sulla strada dritta $\langle\mathrm{sp}\rangle$ $\#<$ p2F\#28> e

Le tabelle 6 e 7 confrontano e accorpano i dati sulla costruzione presentativa ricavati dall'esame del CLIPS e del LIP. La tabella 6 presenta il dato sulle occorrenze espresso in termini di numerosità assoluta e come percentuale sul totale delle occorrenze registrate. La tabella 7 dà un'indicazione sulla frequenza del costrutto nei campioni: tale valore è ricavato calcolando la percentuale di parole grafiche che nei due corpora sono utilizzate in costrutti presentativi.

Tabella 6

$\begin{array}{llrrrrr} & & \text { Firenze } & \text { Milano } & \text { Roma } & \text { Napoli } & \text { Totale } \\ \text { CLIPS } & \text { c'è presentativo } & 2 & 2 & 5 & 7 & 16 \\ \text { LIP } & \text { c'è presentativo } & 38 & 24 & 42 & 26 & 130 \\ \text { Totale } & \text { c'è presentativo } & 40 & 26 & 47 & 33 & 146 \\ & \% & \mathbf{2 7 , 4} & \mathbf{1 7 , 8} & \mathbf{3 2 , 2} & \mathbf{2 2 , 6} & \mathbf{1 0 0}\end{array}$




$\begin{array}{llrrrrr} & & \text { Firenze } & \text { Milano } & \text { Roma } & \text { Napoli } & \text { Totale } \\ \text { CLIPS } & \text { p.g. } & 5917 & 5002 & 4604 & 3787 & 19310 \\ \text { LIP } & \text { p.g. } & 125000 & 125000 & 125000 & 125000 & 500000 \\ \text { Totale } & \text { p.g. } & 130917 & 130002 & 129604 & 128787 & 519310 \\ & \text { p.g in c'è presentativo } & 333 & 216 & 391 & 274 & 1214 \\ & \text { \% } & \mathbf{0 , 2 5} & \mathbf{0 , 1 7} & \mathbf{0 , 3 0} & \mathbf{0 , 2 1} & \mathbf{0 , 2 3}\end{array}$

Nel complesso, i dati presentati nelle tabelle 3-7 ribadiscono la bassa frequenza del costrutto presentativo nei corpora esaminati e allo stesso tempo mostrano tracce di differenziazione diatopica, specialmente per quanto riguarda la città di Milano. Nell'interpretazione del dato sulla diatopia, va tenuto però presente che il numero di occorrenze registrate nei campioni è complessivamente basso e che, pertanto, le differenze osservate potrebbero riflettere le caratteristiche dei corpora utilizzati piuttosto che gli usi linguistici dei parlanti di una certa varietà regionale. Il problema della diatopia sarà ripreso più avanti nel paragrafo dedicato alla discussione dell'analisi variazionale (§5).

\subsection{Variabilità diamesica}

Un primo dato sulla variazione diamesica emerge dal confronto del numero di occorrenze presenti nei corpora LIP e CODIS. Come si ricorderà, nel primo sono state trovate 127 frasi con c'è presentativo, mentre nel secondo 116. Il confronto tra i numeri assoluti delle occorrenze registrate nel LIP e nel CODIS è però poco informativo e non permette di evidenziare eventuali differenze nella diffusione diamesica del costrutto esaminato, poiché dal punto di vista dell'ampiezza i due corpus differiscono fortemente. Pertanto si è cercato di ridimensionare i dati dei due corpora rapportandoli ad una dimensione di 100.000 parole, in modo da poter confrontare i dati in modo più diretto. In questo modo troviamo $26(25,9)$ costruzioni per il LIP (ossia una percentuale di 0,026\%) e 0,5 per il CODIS (ossia una percentuale di $0,0005 \%$ ). Tale ridimensionamento dà un'idea indicativa della frequenza del fenomeno nei due campioni: benché generalmente poco diffusa, la frase presentativa ricorre molto più frequentemente nel parlato del LIP che non nei testi scritti raccolti nel CODIS.

La maggiore frequenza del c'è presentativo nel parlato conferma l'analisi di questo costrutto data da Lambrecht (1994). Come si ricorderà (cfr. §2), la funzione discorsiva attribuita alla frase presentativa è quella di introdurre un referente nuovo nell'universo del discorso, rendendolo disponibile per la predicazione espressa immediatamente dopo dalla clausola (pseudo)relativa. L'introduzione di un referente nuovo nel discorso e la sua promozione a topic non possono però avvenire mantenendo il sintagma referenziale nella posizione che esso avrebbe nella frase canonica: esso deve essere invece isolato in posizione periferica o in una clausola a sé stante. Lambrecht si riferisce a questa condizione indicandola con il nome di Principio di separazione della referenza e del ruolo (PSRR). Tale principio si giustifica sul piano comunicativo, poiché garantisce che l'informazione nuova sia introdotta in piccoli blocchi, assicurando che essa venga presentata e utilizzata in modo cognitivamente vantaggioso. Il PSRR trova applicazione nelle costruzioni con 
dislocazione a sinistra o tema sospeso, in cui il SN topicale compare anticipato, in posizione non canonica e, appunto, nei costrutti presentativi. La struttura biclausale, infatti, consente di separare la funzione referenziale dal ruolo relazionale o grammaticale che il denotato ha come argomento della proposizione. Lambrecht collega esplicitamente il PSRR alle esigenze cognitive che parlante e ascoltatore hanno nel pianificare e decodificare uno scambio verbale; tali esigenze sono attenuate nella parola scritta in virtù del suo carattere maggiormente pianificato. Si può ritenere, quindi, che le diverse esigenze di pianificazione e decodifica dei testi scritti e parlati siano all'origine della maggiore diffusione del costrutto presentativo nel parlato ${ }^{5}$. Questa interpretazione lascia però aperti alcuni interrogativi, in particolare riguardo alla rilevanza del costrutto presentativo come tratto tipico del parlato e all'eventualità che la differenza osservata a livello diamesico possa in realtà riflettere differenze variazionali di altro tipo.

In primo luogo, la percentuale molto bassa di occorrenze nel corpus LIP induce a chiedersi fino a che punto sia giustificato definire il c'è presentativo come un tratto caratteristico del parlato contemporaneo, se si calcola che i 130 casi del LIP corrispondono pressappoco allo 0,026\% delle parole grafiche presenti nel corpus ${ }^{6}$. Ciononostante, dal paragone con le percentuali ancora più esigue nel corpus CODIS, si può concludere che il fenomeno sia marginale nello scritto e che da tale punto di vista si sia autorizzati definirlo come «tipico» del parlato ${ }^{7}$. Inoltre, benché il costrutto presentativo non sia frequente, esso può essere ritenuto «tipico» in virtù della sua rispondenza alle necessità di organizzazione testuale proprie del discorso orale. La scarsa frequenza della costruzione potrebbe inoltre essere il riflesso della sua marcatezza. Rispetto alle altre costruzioni enumerate da Lambrecht come esempi di PSRR, il c'è presentativo isola in principio di frase non un costituente topicale, come avviene ad esempio nella dislocazione a sinistra, ma uno focale, dando luogo ad una struttura diversa e più complessa di quella informativamente non marcata costituita dalla sequenza topic-comment.

In secondo luogo, i corpora considerati nell'analisi non differiscono soltanto nelle dimensioni ma anche nell'articolazione diafasica interna. Nel LIP, infatti, i registri più informali sono più ampiamente rappresentati che non nel corpus CODIS. Non si può escludere, quindi, che la differenza riscontrata tra LIP e CODIS celi una differenza al livello diafasico, ossia una diversa frequenza d'uso della costruzione in contesti linguistici spontanei, meno organizzati e informali e in contesti d'uso organizzati e più o meno formali.

\subsection{Variabilità diafasica}

Come appena accennato, la differenza riscontrata nel paragone tra scritto e parlato può essere integrata da un'analisi della distribuzione del c'è presentativo nei vari contesti d'uso della lingua orale.

\footnotetext{
${ }^{5}$ Alcuni studi recenti hanno fornito prove empiriche a sostegno del PSRR enunciato da Lambrecht, mostrando che i topic introdotti in frasi con dislocazioni a sinistra vengono memorizzati e richiamati più facilmente di quelli introdotti in altre costruzioni in cui riferimento e ruolo non sono separati (si veda Kuzar \& Netz 2010 e Netz, Eviata \& Kuzar 2011).

${ }^{6} \mathrm{La}$ scarsa frequenza del costrutto nel parlato è stata riscontrata anche esaminando le produzioni di giovani di cultura medioalta (Bernini 1991; Bozzone Costa 1991).

${ }^{7}$ Come d'altronde già discusso in Berruto (1985).
} 
Infatti, la frequenza del fenomeno nei vari contesti parlati potrebbe chiarire se il c'è presentativo sia più caratteristico del linguaggio orale spontaneo, meno organizzato o piuttosto di un linguaggio più strutturato, formale ed organizzato.

La forte stratificazione diafasica del corpus LIP può essere sfruttata a questo scopo. Infatti, la tipologia dei testi (A, B, C, D e E) offerti nel corpus permette di disporre i dati in un continuum che va dal discorso dialogico, informale e testualmente meno organizzato al discorso monologico, formale ed organizzato. Il punto estremo d'informalità è rappresentato dai testi in A (conversazioni faccia a faccia con pressa di parola libera) e quello di formalità dai testi in D (monologhi in presenza del destinatario, come lezioni, omelie e comizi). La distribuzione delle costruzioni con $c$ 'è presentativo è fornita nella tabella 8:

Tabella 8

\begin{tabular}{|c|c|c|c|c|c|c|}
\hline & A & B & C & D & $\mathbf{E}$ & Totale \\
\hline $\begin{array}{c}C^{\prime} \grave{e} \\
\text { presentativo }\end{array}$ & $25 / 127$ & $18 / 127$ & $29 / 127$ & $25 / 127$ & $30 / 127$ & 127 \\
\hline & $20 \%$ & $14 \%$ & $23 \%$ & $20 \%$ & $24 \%$ & $100 \%$ \\
\hline
\end{tabular}

I dati della tabella mostrano che, nei limiti del corpus analizzato, la distribuzione del c'è presentativo può essere considerata omogenea nei diversi gradi di formalità. Nonostante si osservi una presenza leggermente minore nei testi della sezione $\mathrm{B}^{8}$, la differenza tra le diverse sezioni non è significativa $(\mathrm{p}=0,389)$. A giudicare da questi dati, dunque, le costruzioni presentative sembrano funzionali alle esigenze di vari registri del parlato: appaiono in tutte le modalità di comunicazione, sia in quelle più spontanee $(\mathrm{A} \mathrm{e} \mathrm{B})$ che in quelle meno spontanee e più pianificate $(\mathrm{C}, \mathrm{D}$ e $\mathrm{E})$.

Questa conclusione coincide con le ipotesi formulate negli studi precedenti menzionati all'inizio del contributo. La costruzione presentativa sembra essere un meccanismo di messa in rilievo al quale si ricorre nel parlato in generale, a prescindere dal registro diafasico.

\section{Confronto tra i risultati e prospettive per lo studio dell'italiano neostandard}

Il quadro appena tracciato ci permette di formulare alcune considerazioni sulla dinamica variazionale dell'italiano neostandard. Partendo dall'insieme dei tratti correntemente inclusi nell'italiano neostandard abbiamo verificato in quale misura la costruzione introdotta dal c'è presentativo fosse presente in un corpus di testi parlati e scritti. Un primo risultato dell'analisi concerne la diffusione del fenomeno nel campione esaminato. Come si è visto, il c'è presentativo è generalmente poco utilizzato: ci si può chiedere, quindi, in che misura esso sia da considerare come tratto effettivamente «tipico» dell'italiano neostandard. I risultati dell'analisi suggeriscono che si tratti piuttosto di un tratto (non

\footnotetext{
${ }^{8}$ I testi della sezione B sono trascrizioni di «scambi comunicativi bidirezionali con presa di parola libera non faccia a faccia» (LIP).
} 
frequentissimo) del parlato, abbastanza omogeneamente diffuso nei registri sia formali che informali. L'analisi ha inoltre registrato un'irregolarità nella diffusione diatopica del fenomeno. Nonostante la costruzione presentativa sia ben attestata in tutte le aree geografiche prese in considerazione, lo è con una frequenza diversa, soprattutto a Milano. La tendenza osservata in questo studio ricorda i risultati di ricerche precedenti sulle tensioni tra italiano standard comune e italiano regionale: proprio riguardo alla diffusione del c'è presentativo Berruto aveva ipotizzato l'esistenza di marcatezze diatopiche, dopo aver osservato l'occorrenza nettamente più bassa di tale costruzione in racconti lombardi e più elevata in quelli emiliano-romagnoli (Berruto 1986; cfr. supra, nota 2). Una tendenza analoga è stata osservata per la dislocazione a destra da Crocco (2010), i cui risultati registrano una presenza di tale costruzione significativamente minore a Milano rispetto a Firenze. Nel caso della dislocazione, la differenza diatopica era stata provvisoriamente spiegata riallacciandosi ai risultati dello studio di Galli de' Paratesi sull'adesione all'italiano normativo a Milano (Galli de' Paratesi 1985). Nel caso della costruzione presentativa, però, le differenze osservate in questa sede non possono essere facilmente attribuite ad un atteggiamento diverso rispetto alla norma. Infatti, il costrutto introdotto dal $c$ 'è presentativo - diversamente dalla dislocazione a destra - non ha subito lo stigma delle grammatiche normative tradizionali e quindi, può essere considerato sostanzialmente «corretto» e «accettabile», anche nello scritto?.

Considerati nel loro complesso, i dati sulla diversa diffusione geografica di alcuni tratti neostandard acquistano maggiore chiarezza se interpretati nel quadro delineato dagli studi sul rapporto tra standard, neostandard nazionale e standard regionali in Italia. In particolare i lavori di Berruto (1987, 2009), Cerruti $(2009,2011)$ e Cerruti e Regis (in corso di stampa) hanno mostrato che il processo di ri-standardizzazione osservabile in italiano ha portato alla formazione di norme regionali caratterizzate da tratti linguistici specifici di ogni macroarea. In questa prospettiva il neostandard può essere visto come una varietà individuata dai tratti, tradizionali o meno, comuni a tutte le regioni. Analizzando alcuni tratti morfosintattici tipici degli italofoni piemontesi, Cerruti $(2009,2011)$ e Cerruti e Regis (in corso di stampa) hanno evidenziato che i tratti standard regionali si riscontrano non solo nel parlato colloquiale e in generale nei registri informali, ma emergono anche in testi modello come quelli giornalistici e nel parlato più controllato delle classi colte. Tali tratti costituiscono quindi una cifra caratteristica della varietà regionale standard. Diversamente dai costrutti esaminati da Cerruti e Regis, le presentative analizzate in questo studio rappresentano un tratto dell'italiano comune, in quanto occorrono, anche se con frequenza variabile, tanto nell'italiano parlato settentrionale (Milano), quanto in quello centrale (Firenze, Roma) e meridionale (Napoli). Rispetto ai tratti morfosintattici del piemontese, le presentative sono quindi un tratto neostandard comune. Tuttavia, i dati sulle presentative raccolti qui e quelli presentati in Berruto (1986), come anche quelli relativi alle

\footnotetext{
${ }^{9}$ Già Berruto (1986) aveva suggerito che nel caso delle costruzioni presentative non sussiste il discorso sulla normatività poiché i costrutti sono considerati grammaticalmente corretti. Ad esempio, enunciati come "V'è degli uccelli che sono privi dell'intestino cieco" sono trattati da Fornaciari (1974: 239-240) come normali casi di "costruzione dei verbi impersonali col plurale".
} 
dislocazioni riportati da Crocco (2010), suggeriscono che la differenziazione regionale dell'italiano possa avvenire non solo a livello qualitativo, manifestandosi cioè come presenza di tratti linguistici specifici con diffusione areale non nazionale (standard regionali), ma anche a livello quantitativo, realizzandosi quindi come maggiore o minore presenza di un tratto per il resto attestato su tutto il territorio italiano.

In conclusione, i risultati dell'analisi indicano in primo luogo che la costruzione presentativa introdotta da $c$ 'è è generalmente poco diffusa. In secondo luogo, l'analisi ha registrato, in accordo con quanto osservato già da Berruto (1986) una distribuzione geografica irregolare e una netta tendenza della costruzione a comparire nel parlato, indipendentemente dal grado di formalità. Anche se dal punto di vista variazionale quest'ultimo dato appare essere quello più solido, nonché quello che trova maggiori riscontri nella letteratura sulle costruzioni presentative in altre lingue europee, il dato sulla differenziazione geografica merita anche, a nostro parere, attenzione: esso infatti suggerisce la possibilità che la ri-standardizzazione si manifesti non solo come realizzazione di varietà standard regionali ma anche come preferenza in una certa area per l'uso di un tratto linguistico comunque attestato su tutto il territorio.

\section{Conclusioni}

Come dato di fondo è confermato che le costruzioni introdotte da c'è presentativo appaiono con maggiore frequenza nei testi orali e che pertanto il costrutto può essere considerato un fenomeno del parlato. In particolare, esso si presenta come una risorsa per distribuire in maniera cognitivamente vantaggiosa per parlante e ascoltatore l'informazione nuova, presentandola in due clausole distinte. Ciononostante, va ricordato che la ricorrenza complessivamente bassa in termini di frequenza relativa anche nei campioni di parlato esaminati ne mette globalmente in questione la qualità di costrutto tipico dell'orale. Per quanto concerne i processi di ri-standardizzazione che interessano la lingua italiana, i risultati della distribuzione diatopica e diafasica contribuiscono ad illustrare la dinamica variazionale interna all'italiano. In linea con studi precedenti, evidenziano una tensione tra italiano neostandard comune e standard regionali, poiché la diffusione del costrutto esaminato non risulta completamente uniforme in tutte le aree geografiche prese in esame. A livello diafasico, invece, il costrutto appare distribuito in modo omogeneo nel campione. Infatti, il c'è presentativo appare in tutti i contesti d'uso del parlato italiano esaminati, quindi non solo in quelli diafasicamente definiti come «usi comuni», ma anche negli usi parlati più formali.

Dato questo quadro, l'inclusione del costrutto presentativo con c'è tra i tratti tipici del neostandard sembra piuttosto poggiare sul carattere tipicamente orale della costruzione che non sulla sua marcatezza diafasica o sulla sua ridotta accettabilità dal punto di vista normativo: come si è visto, infatti, non si tratta di un costrutto tradizionalmente stigmatizzato e quindi riaccettato in una fase recente di ri-standardizzazione, ma di un costrutto, che ha le sue radici nelle fasi più antiche dell'italiano (Salvi 2010), globalmente trascurato dalle grammatiche (Berruto 1986). Si tratterebbe 
quindi di un tratto innovativo solo nel senso che esso acquisisce visibilità, anche se non una diffusione quantitavamente importante, in seguito al formarsi, nel periodo postunitario, di varietà parlate di italiano.

\section{Riferimenti}

Ashby, W.J. (1995): French presentation structure, in: Amastae, J., G. Goodall, M. Montalbetti \& M. Phinney (a cura di): Contemporary research in Romance linguistics. Amsterdam, John Benjamins, pp. 91-104.

Albano Leoni, F. (a cura di) (2006): Il corpus CLIPS. Presentazione del progetto. http://www.clips.unina.it.

Alfonzetti, G. (2002): La relativa non-standard: italiano popolare o italiano parlato? Centro studi filologici e linguistici siciliani. Università di Palermo.

Aureli, M. (2003): Pressione dell'uso sulla norma. Le relative non standard nei giudizi degli utenti. Studi italiani di linguistica teorica e applicata, XXXII, 1, pp. 45-67

Aureli, M. (2005): Le relative non standard nel parlato. Tesi di Dottorato, Università di Firenze.

Aureli, M., M. Cennamo, C. Crocco \& F.M. Dovetto. (2005). Aspetti morfosintattici del dialogo, in: Albano Leoni, F. \& R. Giordano (a cura di): Italiano Parlato. Analisi di un dialogo. Napoli, Liguori, pp. 119-135.

Béguelin, M.-J. (2003): Variations entre macro- et micro-syntaxe: de quelques phénomènes de grammaticalisation, in: A. Scarano (a cura di), Macro-syntaxe et pragmatique. L'analyse linguistique de l'oral. Roma, Bulzoni, pp. 111-131

Bernini, G. (1991): Frasi relative nel parlato colloquiale, in: Lavinio, C. \& A. A. Sobrero (a cura di), La lingua degli studenti universitari. Firenze, la Nuova Italia, pp. 165-187

Berretta, M. (1995): Come inseriamo elementi nuovi nel discorso/1: 'C'è il gatto che ha fame', Italiano e Oltre, 53, pp. 79-105

Berretta, M. (1995a): Ordini marcati dei costituenti maggiori di frase: una rassegna. Linguistica e filologia, 1, pp. 125-170.

Berruto, G. (1985). Per una caratterizzazione del parlato: litaliano parlato ha un'altra grammatica?, in Holtus, G. \& E. Radtke (a cura di): Gesprochenes Italienisch in Geschichte und Gegenwart. Tübingen: Narr, pp. 120-53.

Berruto, G. (1986): Un tratto sintattico dell'italiano parlato: il c'è presentativo, in: Lichem, K., E. Mara \& S. Knaller (a cura di.): Parallela 2. Aspetti della sintassi dell'italiano contemporaneo. Tübingen: Narr, pp. 61-73.

Berruto, G. (1987): Sociolinguistica dell 'italiano contemporaneo, Roma, La Nuova Italia Scientifica.

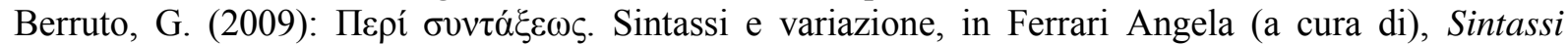
storica e sincronica dell'italiano. Subordinazione, coordinazione, giustapposizione, vol. I, Firenze: Cesati, pp. 21-58.

Bonomi, I. (2002): L'italiano giornalistico. Dall'inizio del '900 ai quotidiani on line. Firenze, Cesati.

Blasco Ferrer, E. (2004): Tipologia, storia e classificazione delle costruzioni presentative romanze. Contributo a una teoria della grammaticalizzazione. Quaderns de filología. Estudis lingüístics, 9, pp. 27-49

Bozzone Costa, R. (1991). Tratti substandard nel pallato colloquiale, in: Lavinio, C. \& A. A. Sobrero (a cura di), La lingua degli studenti universitari. Firenze, la Nuova Italia, pp. 123-163.

Cerruti, M. (2009): Strutture dell'italiano regionale. Morfosintassi di una varietà diatopica in prospettiva sociolinguistica. Frankfurt am Main: Peter Lang.

Cerruti M. (2011): Regional Varieties of Italian in the Linguistic Repertoire.International Journal of the Sociology of Language, 210, pp. 9-28.

Cerruti, M. (in corso di stampa): Varietà dell'italiano, in: G. Iannaccaro (a cura di), La linguistica italiana all'alba del terzo millennio (1997-2010), Roma, Bulzoni.

Cerruti, M. \& R. Regis (in corso di stampa): Standardization Patterns and Dialect/Standard Convergence: A North-Western Italian Perspective. In: Language in Society, 43, 1. 
Cinque, G. (1988): La frase relativa, in: Renzi, L, G. Salvi \& A. Cardinaletti (a cura di): Grande grammatica italiana di consultazione, Bologna, Il Mulino, vol. 1, pp. 443-503.

Crocco, C. (2010): La dislocazione a destra tra italiano comune e variazione regionale», in: Pettorino, M., A. Giannini \& F.M. Dovetto (a cura di): La Comunicazione Parlata 3. Università degli studi di Napoli l'Orientale.

Cruschina, S. (2012): Focus in existential sentences, in: Bianchi, V. \& C. Chesi, (a cura di), Internet celebration for Luigi Rizzi's 60th birthday, pp. 1-31.

D’Achille, P. (1990): Sintassi del parlato e tradizione scritta della lingua italiana. Analisi di testi dalle Origini al secolo XVIII. Roma, Bonacci.

De Cesare, A.M. (2006): C'è la tua bambina che gioca coi fiammiferi. Funzioni del costrutto presentativo c'è... che ...., in: Ferrari, A. (a cura di): Parole frasi testi tra scritto e parlato (Cenobio 55/3), 2006, pp. 215 - 221.

De Cesare, A.M. (2007): Sul cosiddetto 'c'è presentativo'. Forme e funzioni, In De Cesare, A.M. \& A. Ferrari (a cura di), Lessico, grammatica, testualità (ARBA 18), Basel, University of Basel, pp. 127-153.

De Cesare, A.M. (2010): Gli impieghi di ecco nel parlato conversazionale e nello scritto giornalistico, in: Ferrari, A. \& A.-M. De Cesare (a cura di): Il parlato nella scrittura italiana odierna. Riflessioni in prospettiva testuale. Frankfurt a/M, Peter Lang, pp. 105-147.

De Mauro, T. (1963): Storia linguistica dell'Italia unita. Bari, Laterza.

De Mauro, T., F. Mancini, M. Vedovelli \& M. Voghera (1993): Lessico di frequenza dell'italiano parlato. Roma, Etas libri,. http://badip.uni-graz.at/.

Fornaciari, R. (1974): Sintassi italiana. Firenze, Sansoni. [ristampa dell'edizione anastatica del 1881, con presentazione di G. Nencioni].

Galli de' Paratesi, N. (1985): Lingua toscana in bocca ambrosiana. Tendenze verso l'italiano standard: un'inchiesta sociolinguistica. Bologna, Il Mulino.

Choi-Jonin, I. \& V. Lagae (2005): Il y a des gens ils ont mauvais caractère. A propos du rôle de il y a, in : A. Murguía (a cura di) : Sens et Références. Mélanges Georges Kleiber, Tübingen, Narr, 39-66.

Davidse, K. (1999): Are there sentences that can be analyzed as there-clefts?, in: Tops, G., B. Devriendt \& S. Geukens (a cura di): Thinking English grammar: to honour Xavier Dekeyser. Leuven/Paris, Peeters, pp. 179-195.

Kuzar, R. \& H. Netz (2010): Empirical support for the principle of the separation of reference and role. Journal of Pragmatics 42, pp.1460-1468.

Lambrecht, K. (1988): Presentational cleft constructions in spoken French, in: Haiman, J. \& S. A. Thompson (a cura di): Clause combining in grammar and discourse. Amsterdam, John Benjamins, pp. 135-179.

Lambrecht, K. (1994): Information structure and sentence form. Cambridge, Cambridge University Press.

Lambrecht, K. (2001): A framework for the analysis of cleft constructions. Linguistics 39, pp. 463561.

Lombardi Vallauri, E. (2011): Focalizzazioni. In: Simone, R, G. Berruto \& P. D’Achille (a cura di), Enciclopedia dell'italiano, Roma, Treccani.

Mioni, A. (1983): Italiano tendenziale: osservazioni su alcuni aspetti della standardizzazione, in: AA.VV.: Scritti linguistici in onore di Giovan Battista Pellegrini, 2 voll. Pisa, Pacini, pp. 495-517.

Netz, H., Z. Eviatar \& R. Kuzar (2011): Do marked topics enhance memory? Research in Language 9 (2), pp.5-17.

Panunzi, A. (2005): Essere e esserci nella lingua italiana d'uso. Indagine su un corpus di parlato spontaneo e primi confronti interlinguistici nelle lingue romanze, in: Korzen, J. (a cura di): Lingua, cultura e intercultura: l'italiano e le altre lingue. Copenhagen Studies in Language 31. Copenhagen, Samfundslitteratur Press, pp. 255-266.

Radford, A. (1975): Pseudo-relatives and the unity of Subject Raising. Archivium Linguisticum, 6:3264.

Roggia, C.E. (2008): Frasi scisse in italiano e francese orale: evidenze dal C-ORAL-ROM, Cuadernos de filologia italiana 15 , pp. 9-29.

Roggia, C.E. (2008a): Le frasi scisse in italiano. Struttura informativa e funzioni discorsive. Genève, Slatkine. 
Rossini Favretti, R. (2000): Progettazione e costruzione di un corpus di italiano scritto: CORIS/CODIS, in: R. Rossini Favretti (a cura di): Linguistica e informatica. Multimedialità, corpora e percorsi di apprendimento. Bulzoni, Roma, pp. 39-56. http://dslo.unibo.it/coris_ita.html.

Sabatini, F. (1985): L'«italiano dell'uno medio»: una realtà tra le varietà linguistiche italiane, in: G. Holtus e E. Radtke (a cura di): Gesprochenes Italienisch in Geschichte und Gegenwart. Tübingen, Gunter Narr Verlag, pp. 155-184.

Sabatini, F. (1980): Linee di tendenza dell'italiano contemporaneo e problemi di norma, in AA.VV. (a cura di): La lingua italiana in Finlandia. Atti del primo convegno degli insegnanti di italiano in Finlandia. Turku, pp. 73-91

Sabatini, F. (1982): La comunicazione orale, scritta e trasmessa: la diversità del mezzo, della lingua e delle funzioni, in: Boccafurni, A.-M. \& A. Serromani (a cura di): Educazione linguistica nella scuola superiore: sei argomenti per un curricolo. Roma, Provincia di Roma, pp. 105-127.

Salvi, G. (2010): Frasi presentative, in: Salvi, G. \& L; Renzi: Grammatica dell'italiano antico. Bologna: Il Mulino, vol. I, pp. 173-178.

Serianni, L. (2000): Italiano. Torino, Garzanti.

Simone, R, G. Berruto \& P. D'Achille (a cura di): Enciclopedia dell'italiano. Roma, Treccani.

Sobrero, A.A. (1992): L'italiano di oggi, Istituto della Enciclopedia Italiana Treccani, Roma.

Sornicola, R. (2007): Strutture relative non standard di varietà parlate: un confine problematico tra modificazione e predicazione, in: F. Venier (a cura di): Relative e pseudorelative tra grammatica e testo. Alessandria, Edizioni dell'Orso, pp. 99-116.

Speelman, S. (1997): ABUNDANTIA VERBORUM. A computer tool for carrying out corpus-based linguistic case studies, Tesi di dottorato, Katholieke Universiteit Leuven.

Venier, F. (2002): La presentatività. Sulle tracce di una nozione. Alessandria: Edizioni dell'Orso.

Venier, F. (2004): L'articolazione semantico-pragmatica dell'enunciato nella didattica dell'italiano. Studi di grammatica italiana, XXIII, pp. 191-237.

Venier, F. (a cura di) (2007): Relative e pseudorelative tra grammatica e testo. Alessandria: Edizioni dell'Orso.

Venier, F. (2007): Introduzione. Convergenze e divergenze tra grammatica e testo, in: Venier, F.: Relative e pseudorelative tra grammatica e testo. Alessandria: Edizioni dell’Orso, pp. 7-30. 